\title{
Papel de la Educación en la prevención de la criminalidad femenina
}

\section{Autora: Yeriny del Carmen Conopoima Moreno}

Doctora en Ciencias. Universidad Metropolitana. Matriz Guayaquil. República del

\section{Ecuador.}

\section{ORCID: https://orcid.org/0000-0001-9998-3681}

Email: conopoima@umet.edu.ec

Fecha de recepción: 23 de enero de 2020

Fecha de aceptación: 05 de abril de 2020

\section{Resumen}

Reflexionar en torno a las causas que impulsan la comisión de delitos, es ubicarse en un amplio espectro de factores, no obstante, se ha determinado que dentro de esos factores se cuentan el nivel socioeconómico de quienes incursionan en acciones criminales, que a su vez se vincula directamente con la educación, la cultura, la familia, la sociedad, la psicología, y la economía del individuo. Dentro de ese campo delictivo, la criminalidad femenina, aun cuando se ha ido progresivamente incrementando, es un tema que pareciera no tener la importancia que merece. De hecho, se realizan diversidad de estudios alrededor de la temática delictiva masculina, pero en cuanto a la femenina, no pareciera despertar el mismo interés. De allí que el presente artículo abordó esta importante problemática de la criminalidad femenina desde el rol que debe cumplir la educación para su prevención. Se partió de la premisa de la Escuela como factor de protección en las conductas transgresoras y como elemento coadyuvador de la educación que debe ser impartida en la familia para una formación en valores.

Palabras clave: Escuela. Prevención, Criminalidad Femenina

\footnotetext{
Abstract

To reflect on the causes that drive the commission of crimes, is to be located in a wide spectrum of factors, however, it has been determined that within those factors the socioeconomic level of those

Esta obra se comparte bajo la licencia Creative Common Atribución-No Comercial 4.0 International (CC BY-NC 4.0)

Revista Trimestral del Instituto Superior Universitario Espíritu Santo 
who incur in criminal actions is counted, which in turn It directly links with education, culture, family, society, psychology, and the economy of the individual. Within this criminal field, female crime, even though it has been progressively increasing, is an issue that seems not to have the importance it deserves. In fact, a variety of studies are carried out around the male criminal theme, but as for the female one, it does not seem to arouse the same interest. Hence, this article addresses this important issue of female crime from the role that education must play for its prevention. It is based on the premise of the School as a factor of protection in transgressive behaviors and as an auxiliary element of education that must be taught in the family for training in values.

Keywords: School. Prevention, Female Criminalit

\section{Introducción}

A través del tiempo, se han desplegado diversidad de sucesos que han hecho historias en el mundo, desde distintos ámbitos como el político, económico, religioso, social y educativo. No obstante, dentro de ese bagaje de eventos, surge un tema que se destaca dentro de la realidad nacional e internacional, y es el referido a la situación de la violencia, situación que forma parte de la realidad de las distintas sociedades alrededor del planeta, y dentro de esa violencia, está presente como una forma de expresión de la misma, la criminalidad femenina.

La delincuencia femenina a lo largo de la historia, se ha desarrollado de forma más tímida que la masculina, pero al observar con detenimiento este fenómeno, se detecta un aumento de la participación de este género, en épocas recientes en acciones criminales. Heimer y Kruttschnitt, (2006), comentan que es a partir de la década de los años setenta, que surge principalmente en los países anglosajones una significativa producción bibliográfica sobre la criminalidad femenina y un creciente interés por desentrañar las complejas relaciones entre género y delito.

Para Serrano y. Vásquez, citado por Sánchez (2012), la delincuencia femenina, a lo largo de la historia ha sido siempre mucho menor que la masculina, pero al analizar detenidamente este fenómeno, se ha observado un aumento en el índice delictivo femenino en las últimas décadas. A pesar del incremento de la criminalidad femenina, tanto a nivel teórico como en la práctica de investigaciones científicas dentro del campo de las ciencias penales, son pocos los estudios 
orientados a identificar las causas o el origen de este problema.

Por su parte, Heimer and Kruttschnitt, (2006), comentan que es a partir de la década de los años setenta, que surge principalmente en los países anglosajones una significativa producción bibliográfica sobre la criminalidad femenina y un creciente interés por desentrañar las complejas relaciones entre género y delito. Antony, (2007), señala que, en el caso de América Latina, la existencia de estudios sistematizados sobre la evolución de la delincuencia femenina es aún más limitada, lo que evidencia una atención de mayore proporciones hacia la criminalidad masculina, en comparación con la femenina.

La poca emisión de material investigativo alusivo acerca de la criminalidad protagonizada por el género femenino, demuestra el carácter secundario que se ha imprimido a esta problemática, dejando a un lado la relevancia que merece, vale decir, sin otorgarle la significancia que representa, dejando claro cómo se marca la hegemonía del varón incluso en el plano de la criminalidad, dejando entrever el poco interés por conocer las causas que han llevado al aumento considerable de actividades delictivas por este sector de la población, dejando también de lado la creación de planes de abordaje para su atención.

En líneas generales, puede decirse, que generalmente el protagonista de los hechos criminales a través de los años, había sido el hombre; sin embargo, al irse expandiendo la actividad delictiva en las mujeres, en delitos que parecían ser de exclusividad masculina, ha llamado la atención hacia la criminalidad femenina, más aun cuando en sus inicios la participación de estas estaba concentrada hacia cierta categoría de delitos y actualmente se le ha visto incursionar en crímenes de mayor gravedad.

Este crecimiento gradual de la incursión delictiva por parte de las mujeres, ha llevado a buscar las razones que le han impulsado a hacerlo, dentro de las posibles explicaciones, se encuentran el consumos de alcohol o drogas, el maltrato dentro de la familia, bajo nivel de escolaridad, los problemas de índole económica, la falta de oportunidades laborales bien remuneradas, el dejarse llevar por el medio circundante o por seguir a su pareja; sin embargo, se viene asociando este fenómeno a la liberación femenina, sobre todo en el campo económico, sin embargo, uno de los principales problemas para precisar la magnitud del problema, son las pocas investigaciones e información que se han realizado en torno al tema. 
Por otra parte, tal como lo señalan Storvoll y Wichstrom, (2002) la falta de supervisión, la crianza sobreprotectora, y el bajo apego a los padres se han señalado como factores que afectan especialmente la conducta antisocial de las hembras. Además, partiendo del hecho de permanecer con mayor regularidad en el hogar, el repetirle constantemente la importancia de la familia, el no estar reunidas por tiempo significativo con compañeros conflictivos y el tener mayores relaciones afectivas en el entorno que las rodea, se convierte en factores protectores ante la posibilidad de incursionar en transgresiones.

Yugueros (2013, pág. 315) por su parte, expone muy sintéticamente las causas por las cuales las mujeres delinquen, señalando que son debidas mayoritariamente a la pobreza, exclusión social, falta de instrucción educativa y otras circunstancias socioculturales. Sin embargo, no deja de ser signo de preocupación el hecho que este fenómeno se haya hecho muy evidente, y que a pesar que proporcionalmente, exista menos mujeres delincuentes que hombres, ello ha de ser objeto de análisis, puesto que existe una realidad palpable y es que no se le ha dado la relevancia que merece al no ser estudiado de la misma manera que la delincuencia masculina, quedando la delincuencia femenina como un tema siempre olvidado.

Es esencial señalar, que existe el error al establecer que la participación de la mujer en actos delictivos pasa por una situación poco esencial o intrascendente, apoyar ello sería una falta de percepción sobre un problema social que viene incrementándose de forma paulatina. Todo ello lleva a sostener la importancia y obligatoriedad de volver la mirada hacia la conducta infractora de las mujeres, lo que debe implicar una intervención activa en corto plazo y todos los ámbitos de actuación y una mejor reflexión para tener una comprensión cabal de los fenómenos asociados a la inserción femenina en actos delictivos.

Cabe mencionar, que la criminalidad femenina ha tomado realce al incrementarse en las sociedades su participación, por lo tanto, importa analizar la incidencia de la familia como factor de predicción de conductas antijurídicas, cuando deja de ser un elemento protector. No obstante ser la familia el primer medio de control social, por ser allí donde el niño aprende a socializar positivamente. Un fracaso en esa etapa lleva a los problemas sociales que hoy se observan diario, como el uso de la violencia para resolver conflictos o la inexistencia de valores como la responsabilidad, la solidaridad o el respeto de límites. 
Se ha manejado con mucha fuerza, la idea que la formación académica es función exclusiva de la escuela y la formación en valores le corresponde a la familia; sin embargo, no puede perderse de vista, que la realidad que está presente en la actualidad, exige con fuerza tomar en cuenta no solo a la escuela o la familia como responsables de la configuración de la conducta de las personas, ha de considerarse adicionalmente, su entorno, puesto que del vínculo que establecen estos tres factores, se va a conformar la conducta futura; lo que demuestra la importancia que se desprende de su trabajo en conjunto.

Partiendo de lo anteriormente expuesto, cabe referir entonces, que la familia se convierte en el primer medio de desenvolvimiento del ser humano, vale decir, que es el primer espacio de contacto del niño o niña, y por ende las experiencias que desarrolle en su núcleo, serán determinante en la conformación de su personalidad, por lo tanto es imprescindible, tener en cuenta la importancia de desarrollar un clima de armonía y afecto, sin obviar la disciplina o el principio de autoridad, que no significa en ningún momento el maltratarlo, sino brindarle las herramientas para desarrollar, su autoestima, confianza, respeto, honestidad, solidaridad, sinceridad, por mencionar algunos. No puede obviarse, que la familia se constituye en el primer modelo o patrón a implantarse en los hijos, sea este pleno de principios o carentes de valores.

\section{Desarrollo}

Evidentemente, el grupo familiar, tiene tres aspectos básicos que cumplir en su acción tutelar: el aspecto material, educativo y afectivo, estos surgen y funcionan cuando los padres tienen elementos para actuar como guías y como prototipos humanos a quienes hay que admirar y seguir en su ejemplo. Pero en innumerables casos, los padres no cumplen con las funciones antes mencionadas, sino por lo contrario, la propia estructura familiar, crea un clima perturbador, que, actuando en la época del crecimiento, provoca graves alteraciones en la personalidad de los hijos, que los pueden llevar a la consumación de los actos ilícitos, situación de la que no están exentas las féminas

No es un secreto para nadie, la importancia de la educación en el desarrollo del ser humano, no obstante, también es u hecho indiscutible, que la formación académica hoy día ha dejado de ser 
un símbolo de progreso, de crecimiento personal, o una forma de mejorar las condiciones de vida, pero lo cierto es que si no existe una educación de calidad, se estará inmerso en una sociedad, sin justicia, ni igualdad, porque se van acortando las posibilidades de acceso a mejores condiciones de vida, lo que a su vez va reduciendo las oportunidades de buenos ingresos, abriendo lamentablemente como única vía de superación rápida la actividad delictiva. Es así como se ha incrementado la criminalidad femenina, erigiéndose como problema social que implica riesgo para la seguridad ciudadana y además se constituye en emblema para obtener objetos materiales.

Sin duda, la educación, el trabajo y vivir dignamente, son derechos universales inalienables, los cuales son deber del Estado, proveerlos, ya que de su existencia depende el desarrollo de un país. Desafortunadamente, estos derechos no están presente de manera equitativa en el mundo, y se da el caso de la dificultad de acceder a la escuela, lo que a su vez limita las posibilidades de empleo, redundando ello en la limitación de ingresos y por ende en la calidad de vida de las personas, pudiendo ser esto el detonador de la participación criminal femenina.

Esta situación es indicativa de la existencia de graves problemas en el área educativa como formadora de hombres y mujeres, y debería ser el motor para actuar sobre la situación de estancamiento que padece la educación, llevando a generar planes para atacar el abandono que en muchos casos padece el sector educativo, que por supuesto confluye no solo en una educación inidónea, sino también en la falta de los talentos que van a desarrollar el progreso de un país.

En efecto, la familia y la escuela constituyen factores de socialización, la familia porque brinda aprendizajes básicos necesarios para el desenvolvimiento autónomo dentro de la sociedad, tales como valores, lenguaje, control de la impulsividad, entre otros y la escuela transmite la necesidad de ser personas honestas y responsables, completando la labor que se realiza en el seno del hogar. Ahora bien, los niños y niñas, que asisten con regularidad a las aulas de clase, y se destacan académicamente, por lo general, no se reúnen con aquellos, que pueden tentarlos o impulsarlos a incurrir en actos contrarios a la norma; ello posiblemente parte del hecho que la escuela se convierte en un factor de protección para los escolares, puesto que al estar en actividad académica, están ocupado en algo productivo; en caso contrario, cuando los niños, niñas y adolescentes, están involucrados en ocio la mayor parte del tiempo, las conductas transgresoras se hacen presente en ellos, con mayor regularidad. 
Podría inferirse de lo señalado anteriormente, que los niños, niñas y adolescentes, menos capacitados y con conducta rebelde, desobediente, en la escuela tienen más probabilidades de cometer delitos que aquellos que presentan un buen rendimiento escolar y un buen ajuste en el colegio, lo que aunado al hecho de carecer de una buena educación en valores, implica que estas infantes y jóvenes, se conviertan objetivos fáciles para enrolarse con personas inescrupulosas que les incite a participar en delitos.

Brunner (2005), con respecto a la relación entre déficit educacional y actividad delictiva, lleva a recoger tres argumentos vitales para explicar tal correspondencia diciendo que en primer lugar para Lochner y Moretti, (2001). la mayor escolarización aumenta la posibilidad de que las personas se ocupen en profesiones legítimas, razón por la cual, de incurrir en conductas ilegales cuya consecuencia fuera la neutralización en cárcel, por ejemplo, se estaría perdiendo ingresos laborales elevando el costo de oportunidad de la delincuencia y el costo del tiempo pasado en prisión. En segundo lugar, Brunner (2005), destaca que esta correlación se explica en una especie de efecto civilizatorio, según el cual la educación modifica pautas culturales alterando, aumentando, la aversión a los comportamientos contra el orden social y, en tercer lugar, sencillamente, se ha dicho que el hecho de ocupar el tiempo en estudiar reduce las posibilidades físicas de delinquir.

De igual manera la Organización de las Naciones Unidas, (Ecosoc, 2002), brinda una serie de políticas para la prevención del delito, destacando el desarrollo socioeconómico, la inclusión social, la salud y la educación, entre otras, fundamentando la prevención del delito desde fuera del sistema penal, entendido esto como cuerpo de medidas y estudios que comprenden una amplia gama de objetos que no se centran en la utilización del sistema penal para prevenir el delito haciendo precisamente énfasis en la educación, el empleo, la familia, la salud o diferentes políticas sociales.

Es importante mencionar, que los niños que proceden de hogares donde prevalece una dinámica conflictiva, en líneas generales no tienen un buen rendimiento, ya que si existe maltrato, problemas económicos, consumos de alcohol o drogas, abandono, ausencia de afecto, por mencionar algunos, lejos de estar motivados para obtener altas calificaciones, más bien se encuentran sumergidos en frustraciones, que le impulsan a despreocuparse por las actividades 
escolares e incluso tienden a abandonar las aulas, siendo presa fácil del medio que le circunda y que pueden inducirle a cometer actos reñidos con la ley.

Esta situación en la actualidad afecta por igual a varones y hembras, echando por tierra las ideas que habían prevalecido por muchos años, donde se afirmaba la prevalencia de la conducta criminal masculina, esto producto del hecho que las tradiciones científicas y sociales han construido como exclusiva problemática del varón, la comisión de hechos delictivos; esto había hecho germinar el pensamiento de asociación de crimen con la población masculina, lo que a su vez dio lugar que la criminología ignorara a la mujer como autora de transgresiones. Sin embargo, en los últimos tiempos, el número de mujeres incursas en delitos se ha incrementado notablemente.

Los criminólogos han estudiado durante mucho tiempo la relación entre las variables relacionadas con la escuela y la delincuencia; ante ello, Lotz y Lee (1999), indican que la escuela es junto a la familia, el contexto más importante y con más influencia en el desarrollo social e individual de los niños, niñas y adolescentes; no sólo se aprenden normas para una adecuada convivencia en la sociedad sino que se adquieren y se pueden mantener patrones de aprendizaje de diferentes comportamientos, entre ellos conductas antisociales y delictivas. Una multiplicidad de variables relacionadas con la escuela, han sido vinculadas de manera consistente, con la conducta infractora, incluido el compromiso de la escuela, la participación y la asistencia a la escuela, sin obviar el clima escolar.

Jacob y Lefgren (2003) examinan la tasa de delincuencia juvenil en días de asistencia escolar comparada con la tasa de criminalidad cuando la escuela no abre sus puertas, por ejemplo, cuando los maestros se encuentran en sus días de capacitación, reuniones de trabajo, atención a los padres, comisiones de servicio, vacaciones, días feriados, entre otras, siendo esta una razón que excusa la asistencia a la institución educativa. El hallazgo principal de este estudio es que el aumento de la asistencia escolar disminuye la tasa total de delitos contra la propiedad en un 14 por ciento. Esto lleva a considerar que la permanencia de los estudiantes en sus aulas de clase, es factor clave para mantener alejados a los estudiantes de situaciones que los involucren en actos criminales.

En líneas generales, puede afirmarse que la educación, a través del tiempo, se ha destacado 
por su carácter social, adicionalmente, por estar conformada por una serie de proceso formativos, que ha dejado huellas, en distintas épocas e innumerables lugares. Se caracteriza, además, por ser un suceso deliberado, mediante una serie de acciones y procesos, que tienen gran influencia en la formación de las personas, logrando esto a través de una serie de conocimientos y habilidades orientados a brindar las herramientas que permitan a toda persona que se someta al aprendizaje, obtener cambios que le favorezcan en su quehacer social. Por ello, la educación es considerada un derecho humano estableciéndole a la escuela la misión de fomentar la cultura, la formación cívica, el conocimiento científico y tecnológico, a lo que hay que añadir, la posibilidad de aprender y de sembrar valores.

\section{Educación y Criminalidad Femenina}

La participación femenina en actividades criminales, es considerada por muchos de los estudiosos de esta problemática, como compleja, ya que primero que nada se rompe con el paradigma de la mujer dulce, tierna, sumisa, para dar paso a una nueva concepción de la misma, en la cual es temeraria y desalmada, quedando atrás el rol de esposa complaciente, madre abnegada, hija dócil, que parecía encajar otrora en todas las mujeres. Asimismo, lleva a reflexionar, en una mujer privada de libertad, en condiciones deplorables, estigmatizada por su familia y la sociedad, al romper esquemas de comportamientos tradicionales, donde no tenían cabida la idea de una hija, hermana, madre o esposa presa.

Toda esa situación lleva a preguntarse, el origen de la decisión de penetrar el mundo del delito y se manejan múltiples posibilidades, tales como el maltrato, las malas compañías, la pobreza, el consumo de sustancias adictivas, la rebeldía, el enamoramiento de algún sujeto con experiencia delictiva, entre otras; pero esencialmente hay que ubicarse en dos aspectos básicos del desarrollo de las niñas y las adolescentes, como es su entorno familiar y su medio educativo.

En el caso de la familia, porque allí se viven las primeras experiencias de vida, donde los padres ofrecen también las primeras enseñanzas, enseñanzas que marcarán su destino de vida, siendo que los buenos ejemplos, el afecto, el inculcar valores positivos como la honestidad, la paciencia, la generosidad, la gratitud, entre muchos guiarán las decisiones a futuro orientadas a una conducta recta.

En el caso de la escuela, allí se van a reforzar los aprendizajes del hogar, y adicionalmente 
se les ofrecerá el conocimiento en áreas como la matemática, la física, la química, la biología y otras tantas asignaturas, pero también se les orientará como manejarse dentro de una conducta cívica, donde la tolerancia, el respeto por los demás y por las leyes debe prevalecer en su actuación.

No obstante, hay que tener presente como influye el liderazgo del docente, su forma de atender su población estudiantil, de abordar las problemáticas presentes, de decir una palabra a tiempo, un consejo pertinente, recordando que cada niña o adolescente que tenga bajo su dirección académica, tiene característica de ser única y por ende una situación personal única, y una mirada a destiempo, un comentario burlón, un trato discriminatorio, puede dar lugar al desvío de una conducta, lo que le da una significancia especial al rol del maestro, pues tiene en sus manos un material moldeable, que si lo trabaja sin dedicación, vocación y precisión, puede a lo largo del tiempo convertirse en alguien que se mueva en el mundo del delito, dejando lejos a la niña o adolescente que tuvo sueños, pero que no pudo concretarlos porque no tuvo quien oyera sus gritos silenciosos pidiendo atención.

En necesario, por lo tanto, reflexionar, en el rol de la familia y del docente en la conformación futura de la niña y la adolescente, pues esos son espacios fundamentales en este aspecto, sin embargo, no debe olvidarse el papel del Estado, en proveer los medios para ocupar sus tiempos de ocio, así como fomentar el descubrimiento y aprovechamiento de los talentos artísticos, deportivos, académicos para hacer de estas niñas y adolescentes grandes mujeres virtuosas y exitosas.

\section{Resultados}

A continuación, se presentan los resultados de un estudio realizado cuyo objetivo fue analizar el papel de la educación en la prevención de la criminalidad femenina. La metodología empleada se realizó respetando los métodos y procedimientos adecuados al tipo de investigación. El trabajo se apoyó en una investigación de campo, se aplicó un cuestionario contentivo de trece preguntas a 20 docentes, 20 padres y 20 funcionarios del Sistema de Protección de Niños, Niñas y Adolescentes, a objeto de obtener información de interés en torno a la situación planteada. A continuación, se presentan los resultados obtenidos. 


\begin{tabular}{cccc}
\hline Pregunta & $\mathrm{Si}$ & $\mathrm{A}$ & $\mathrm{NO}$ \\
& $\%$ & Veces & $\%$ \\
& & & $\%$
\end{tabular}

Se ha incrementado la comisión de delitos por

100

parte del género femenino

La delincuencia femenina se centra

75

25

fundamentalmente en mujeres muy jóvenes

Los problemas económicos impulsan a la mujer

a delinquir

La Educación es factor preventivo de conductas delictivas en niñas y jóvenes

La familia funcional brinda a las hembras

75

25

herramientas para no incurrir en conducta

transgresora.

Los estudiantes con bajo récord académico son

más propensos a incursionar en conductas

transgresoras.

La asistencia regular a la institución educativa e

factor protector de conductas delictivas en las niñas y adolescentes.

La motivación a obtener una profesión desvía la posibilidad de cometer delitos.

La criminalidad femenina se caracteriza por

participar en delitos menos graves

La tasa de delitos protagonizados por mujeres e: significativamente más baja que el índice criminal masculino 
Considera que la escuela en general maneja

suficientes estrategias preventivas en materia de

delincuencia femenina

Implementar actividades extracurriculares en la:

instituciones educativas contribuirían al descenso de 1

criminalidad femenina.

La permanencia en el sistema educativo protege

a las estudiantes de la posibilidad de formar parte de grupos delictivos
25

50

25

\section{Análisis y Discusión e Resultados}

Vale hacer mención que factores asociados con las relaciones que los jóvenes tienen con amigas y compañeros, así como el abandono precoz de los procesos escolares, son identificados por la Organización Panamericana de la Salud como algunos de los factores de riesgo para la conducta violenta adolescente. Igualmente, hay que tener en cuenta, que las adolescentes, al estar experimentado algunas situaciones dentro de su actividad escolar como repitencia, bajo rendimiento, acoso escolar, rechazo, pueden llegar a ser objeto de incitación o reclutamiento para que participen en hechos criminales.

No puede obviarse, que la preparación educativa o académica, y la consecuente permanencia en las instituciones educativas, tienen un carácter de fundamental, tanto para la experiencia formativa del estudiante, como para la conformación de una persona proba, para la prosecución de estudios superiores y para el desempeño de actividades laborales. Sin duda, las jóvenes que permanecen ligadas a su actividad escolar, que no caen en el juego del abandono o deserción educativa, se alejan de situaciones que pueden desembocar en actos que afectar su salud, seguridad, libertad e incluso su vida, por lo que permanecer en la escuela otorga opciones los protege de conductas de riesgo.

Esta opción de protección y apoyo a las niñas y adolescentes a través de la actividad escolar,

Esta obra se comparte bajo la licencia Creative Common Atribución-No Comercial 4.0 International (CC BY-NC 4.0) 
liderizada por sus maestros y profesores, se erige como factor preventivo de gran magnitud, contribuyendo con la generación de la excelencia tanto académica como comportamental de quienes llevarán las riendas en un corto futuro del desarrollo del país.

\section{Conclusiones}

Es indudable que la escuela, se convierte en factor significativo de preparación de las personas, al ser un espacio donde se brinda información académica y cultural, lo que desemboca en un entramado de conocimientos, para su desenvolvimiento, pero que tiene un valor fundamental para la prevención del delito. Ello lleva implícito, que además de ofrecer contenidos pedagógicos a través de programas educativos, debe incluir una verdadera educación cívica, que lleve a crear una cultura que aparte mujeres y adolescentes de la vía del delito.

No puede descartarse en ningún momento, ni en ningún nivel de educación, la implementación de programas que lleven implícitos la prevención del delito, mediante la generación y puesta en práctica de programas de la cultura de la prevención del delito; de igual forma, es menester, invertir en políticas y programas de capacitación en el lugar de desempeño laboral, para con ello, favorecer e impulsar el avance de las mujeres a todos los niveles y en todos los sectores económicos y que por supuesto, se despierte en ellas el deseo de superación y de practicar todo tipo de profesiones, incluso aquellas que pudieran considerarse no tradicionales o aptas para desempeñarlas una mujer.

\section{Referencias Bibliográficas}

Antony, C. (2007). Estudio sobre violencia de género: las mujeres transgresoras. Panamá: Universitaria de Panamá.

Brunner, J. (2005). Educación y Actividad Delictiva: Evidencia Internacional. Fundación Paz Ciudadana.

ECOSOC (2002). Directrices para la prevención del delito. Resolución 2002/13 del Consejo Económico y Social.

Esta obra se comparte bajo la licencia Creative Common Atribución-No Comercial 4.0 International (CC BY-NC 4.0)

Revista Trimestral del Instituto Superior Universitario Espíritu Santo 
Heimer, K. and Kruttschnitt, C. (2006), Gender and Crime. Patterns in Victimization and Offending. Toronto: Department of Sociology at the University of Toronto

Jacob, B. A., Lefgren, L. (2003), Are idle hands the devil's workshop? Incapacitation, concentration, and juvenile crime, en American Economic Review, 93(5), 1560-1577.

Lochner, L. y Moretti, E. (2001). The effect of education on crime: Evidence from prison inmates, arrests, and self-reports (Working Paper 8605). National Bureau of Economic Research.

Sánchez, E. (2012), Delincuencia Femenina. Trabajo Final de Investigación para optar al Título de Magíster en Estudios Interdisciplinarios de Género. Universidad de Salamanca.

Storvoll, E. y Wichstrom, L. (2002). Do the risk factors associated with conduct problems in addolescents vary according to gender?. Journal of Adolescent

Yugueros, A. (2013). La delincuencia femenina. Una revisión teórica. Madrid: Universidad Complutense de Madrid

Esta obra se comparte bajo la licencia Creative Common Atribución-No Comercial 4.0 International (CC BY-NC 4.0) 